\section{ETHERIZATION AND ASPHYXIA.} BX .JOHN SCOTT, Esq., Shelton, Stafford.

Perfaps the history of medicine presents no instance of an active remedy being brought into extensive use with so little injury resulting from its operation, as the late introduction into practice of vapour of ether. The consideration that it has been extensively used by classes of persons little qualified to judge of any untoward symptoms as they presented themselves, or to regulate its most favourable effects so as to prevent their becoming injurions, speaks strongly in its favour, and proves it to he a safe as well as a powerful agent. But as in some few cases injurious results have been produced, it would be well that every attention should be paid to the phenomena exhibited during the process of inhalation, so that the approach of any dangerous pathological state may be more readily distinguished than it has hitherto been, and care be taken to prevent injuries resulting from it.

With this object, it would be well if the profession generally would discontinue communicating instances of its use, where etherization exhibits nothing but its most ordinary phenomena, except where it has been applied to some new purpose; and communicate, more freely than they have hitherto done, instances of disappointment as to its results, and disagreeable consequences resulting from its employment. It is also important to give a clear account of the process employed, recollecting that it is almost an axiom of scientific communication, at the present day, that it is not sufficient to publish the result, but is especially necessary to state the processes by the aid of which they were obtained. If this were carefully done, the result would be, that we should soon have as correct a pathology of etherization as we have of the action of any established remedy; and it would present another example of the progress of this age, if the therapeutic knowledge was completed in a few months instead of being the labour of generations.

There is one circumstance which appears to have been but little thought of in England, although it has attracted attention in France: that is, the pathological condition produced during inhalation from the vitiated yuality of the air breathed. With the purpose of calling attention to the subject, $I$ send you an abstract of an article which appeared in La Presse on the 3 rd and 7 th of this month. The importance of his view of the subject is perhaps there overrated by $M$. Doyère, the writer, but it is one at least worthy of some consideration. After speaking of the manner in which ether has been administered, and giving a rather dramatic picture of etherization, he says:-

"We see that the vapour of ether has acted thus far only in more $\cdots$ less active conjunction with another agent, terrible in its eff:ets, and to which circumstances, altogether independent of the inventor, the invention, or the administrator, may give : preponderance such as, had it been observed at first, would have stiffed the discovery at its birth, and left no other memorial of the experiments than the memory of irreparable disasters. This agent is asphyxia. We hesitate not to attribute to it the greater part of the accidents which have resulted from etherization, and which have brought down upon it those reproaches of which this great discovery has been the subject. What we wish particularly to call attention to, and consider a matter of great moment, is the distinction between etherization and asphyxia, two conditions which have been too much confounded with each other. The phenomena of the latter are well known, and have been so ever since men hanged and drowned themselves, and have been yet better studied in physiological experiments where animals have been enclosed in nitrogen or hydrogen gas Those of the former are still unknown; for they have never yet been observed isolated from those of asphyxia, by subjecting men or animals to an etherous mixture, containing twentyone per cent. of oxygen. The mixtures of etherous vapour which have been hitherto employed have generally contained less than fifteen per cent. of this gas, and consequently the resulting phenomena have been the combined result of inhalation of ether and deprivation of vital air. Suppose that 100 parts of air are mixed with 100 parts of vapour of ether, the twenty-one parts of oxygen contained in the air will be the entire oxygen of the 200 parts of the mixture, and, consequently, the patient respiring it will be breathing an atmosphere containing, not twenty-one, but ten and a half per cent. of oxygen. This is what actually takes place at a temperature of seventeen centigrammes, $\left(62^{\circ}\right.$ Fahr., $)$ a temperature frequently found in a well-warmed room, not higher than the coolest apartment in summer, and one which the inhaling ap paratus is likely to acquire in the immediate vicinity of the patient and the administrator: much more is this the case when heat is applied to the vessel.

Temperature is therefore an important element in the problem we have to study. When a volatile fluid is enclosed in a vessel, the air enclosed with it is always saturated with its vapour, and the saturating quantity is always the same at the same temperature. But as the temperature changes, the proportions of vapour and air change also; the increase in the proportion of vapour being very great as the temperature becomes elevated. The ratio of increase is one, however, which can be very easily calculated, and the following table presents a view of the proportions of ether held in solution at different temperatures, and the proportions of oxygen contained in the mixture:-

\begin{tabular}{c|c|c|c}
\hline \multicolumn{2}{c|}{ Temperature. } & Ether. & Oxygen. \\
\cline { 2 - 4 } Centigrammes. & Fahr. & Vapour of, to 100 of air. & Per cent. of, in mixture. \\
$0^{\circ}$ & $32^{\circ}$ & 25 & 15 \\
$10^{\circ}$ & $50^{\circ}$ & 37 & 13 \\
$20^{\circ}$ & $68^{\circ}$ & 57 & 9 \\
$30^{\circ}$ & $86^{\circ}$ & 83 & $3 \frac{1}{2}$ \\
$35^{\circ}$ & $95^{\circ}$ & 100 & 0 \\
\hline
\end{tabular}

Had the discovery come into use last summer, during the seventy-six days between the 1st of June and the 15 th of August, then on thirty-nine of these days surgeons would have administered a mixture containing less than five per cent. of oxygen, and on the 5th of July and 5th of August, the vapour of ether would have been administered almost pure.

Now although the difference between etherization and asphyxia may not be so great as $M$. Doyère imagines, yet these are facts worthy of consideration. The inhalation of air so poor in oxygen cannot be without its proper influence, and will at least modify the specific results of the inhalation of ether. Stafford, March, 1847.

\section{CASE OF}

DELIVERY BY PERFORATION AND FXTRAC. TION, IN A CASE OF EXTREME PELVIC DISTORTION.

By EDWARD WM. MURPHY, M.D.,

PROAKSSOR OF MIDWIFRR, UNIVERSITY COLLEGE, LONDON.

(Read before the Westminster Msdical Society, March 27th, 1847.)

The following case of delivery by the crotchet, in extreme distortion of the pelvis, may perhaps interest the members of this Society, as additional facts to assist us in determining the rule of practice in these serious and most difficult cases.

E. B-, aged thirty-three years, born of diminutive parents, was perfectly healthy and well-formed until five years of age. She was then attacked with measles; the ernption suddenly disappeared, and she was seized with inflammation of the chest. During her illness, her parents, from limited means, were obliged to depend on parochial assistance, and from this date the disease commenced that produced her deformity. The account given of its progress is homely, but sufficiently expressive. Her parents say, "she was neglected by the doctor; that she conld not lie down for seven days, from difficulty of breathing, during which time she scarcely passed any water. The disease fell into her ankles; then into her knees, and eventually settled in her back. It remained in her back for seven years before she could get up and down stairs. She was eighteen years old before she could walk half a mile without a stick. She forgets whether there was any sediment in her water during herillness." Such is her history. The deformity that resulted, affected chiefly the spinal column. All the extremities were small, but no other deviation in them than that she was knock-knee'd. There was a curvature in dorsal vertebre strongly to the right side, and the whole of the lumbar vertebra were thrown forwards in extreme convexity, so that the aspect of the pelvis was changed, the axis of the brim of the pelvis being nearly horizontal. Her height was about four feet three inches.

About June last she became pregnant; and the duration of her pregnancy, dated from the cessation of the catamenia, (June 15th,) would be 280 days, to March 22 nd. Unfortunately; for an obvious reason, her pregnancy was concealed, which was more easily accomplished in consequence of the natural prominence of the abdomen, produced by the curvature of the spine. She was taken in labour on Tuesday, March 8th; the 
pains continued for about six hours, when the waters broke. They then ceased for twelve hours, were again renewed, and continued regularly during Wednesday and Thursday, on which day I saw her for the first time.

This patient, living in humble life, resided in Rickmansworth: she placed herself under the care of Mr. Codd, a very intelligent gentleman, practising in that locality. As soon as it became evident to him that the deformity of the pelvis was an obstruction to the delivery of his patient, he requested my assistance, and having accompanied him on Thursday evening, I found her in active labour, her spirits good, and her pulse tranquil. A vaginal examination proved the justice of $\mathrm{Mr}_{2}$. Codd's fears. The pelvis afforded an interesting example of the ovate deformity; there was sufficient space in the outlet and cavity of the pelvis to admit the finger readily; but when it passed upward, the projecting sacrum at once arrested it, and presented such a prominence, that a superficial observer might mistake it for the head of the child. It was necessary to introduce the left hand, in order to make a satisfactory examination, and when the two first fingers were passed upwards and forwards above the pubis, the os uteri was found open to the size of a shilling, soft, dilatable, and free from tenderness, although the head seemed to press it firmly between the pubis and promontory of the sacrum. It was impossible to deter. mine the position of the head; neither sutures nor fontanelles could be reached; a smooth surface of bone (presumed to be the parietal) occupied the mouth of the womb.

The difficulties which this case presented were not diminished by the circumstances in which she was placed, being in a confined apartment and dwelling, in a village sufficiently far from London to render any additional aid impracticable. The state of her labour also rendered it impossible to remove her thither. I therefore gave up the idea of an operation, which then seemed to me the only one available, because the distance between the pubis and promontory of the sacrum was only an inch and a half, and it appeared a very hopeless matter to expect to succeed with the crotchet. Had she been in London, and I had had the support of my colleagues who are eminent in obstetric practice, I think I should have undertaken the Casarian section, employing every precaution for its success, such as has been recently introduced in operations very similar to it; but in Rickmansworth this was out of the question. Perforation of the head was the only operation that could be attempted.

Having explained my views to $\mathrm{Mr}$. Codd, and also to $\mathrm{Dr}$. Ayres and Mr. Garlick, the resident medical gentlemen who were present, I undertook the operation. About seven o'clock P.M. the perforator was applied cautiously to the cranium, and finding that the head did not recede, it was forced through to the stops. An opening was easily made in the transverse axis of the brim; but the blades could not be separated in the conjugate direction. The moment the instrument was withdrawn, the opening closed. It was therefore necessary to reintroduce it, and to keep the finger within the cranium until the crotchet was applied. This was accomplished with no little difficulty. Having at length succeeded, and removed as much of the brain as possible, two fingers of the left hand were placed between the head and os uteri, with the object of guarding the crotchet, as well as dilating, in this artificial way, the os uteri itself. The instrument being thus placed, traction was made with every pain, aud discontinued in the intervals, in order that the patient might have rest, and be saved from the effect of continuous pain when there seemed to be no hope of any immediate termination to her sufferings. In this manner, three hours were spent without any advance of the head being made. The cranium on the left and depending side of the pelvis was broken up completely; and having carefully removed all the fragments, I thought it advisable to cease any further efforts then; to give her an anodyne, so as to secure some rest, and, if possible, sleep. I hoped that then, the action of the uterus being renewed, the head might descend somewhat more within reach. Mr. Codd, who was jresent during the operation, agreed with me in this view, and we left her for the night.

March 12th.-About eight o'clock this morning we returned. She had some sleep during the night, after which the action of the uterus returned; but the pains were less powerful, and at longer intervals than before. 'The pulse had increased in frequency to about 110; in other respects there was no alteration. The operation was resumed. The vagina was still moist and free from tenderness; the os uteri dilated to about one-half, and the broken bones of the cranium protruded through it sufficiently to prove that the head had advanced a little during the night. The attempt was made to replace the crotchet as before; but this could not be done. I then endeavoured to fix it in the foramen magnum: this also failed. Several varieties of craniotomy forceps were tried, for the purpose of seizing the bones firmly. I found it very difficult to use them in so confined a space, and when applied, they only broke away that portion of the bone within their grasp, leaving the remainder of the head unmoved. The fragments of bone were thereby increased, and having carefully removed them, I again returned to the crotchet, placing it in a direction the opposite to that I had used before. The point was now directed to the right side of the pelvis, and fixed within the cranium, close beside the sella turcica; traction was used cautiously; the force was sufficient to break through the orbitar plate of the frontal bone, and thus a firm purchase was obtained. There was no risk now of the instrument slipping; the extractive force was consequently increased gradually to my utmost strength; still I conld not move the head. After the interval of rest, Mr. Codd also tried to do so, but was equally unsuccessful. I endeavoured, in imitation of Johm Clarke, to get the base of the cranium obliquely in the brim of the pelvis, but it made no difference. This was to me an anxious moment, because the obstacle seemed to be in. superable. However, I persevered a little longer, and found that the bone was separating; half the frontal bone was removed; it was broken across at the orbit, and separated at the frontal suture. The crotchet could not now be reapplied within the cranium; I resolved, therefore, before making any further attempt, to allow the patient some time to rest. We left her for some hours, and on our return I found the pulse increased to 120 ; she had no sleep, but complained of constant pain in the lower part of the back; the action of the uterus continued as before; the ragina was becoming a little heated; the head, or rather the fragments of it that remained, had occupied the brim more fully; the mouth of the uterus was quite dilated. I applied the crotchet on the outside of the scalp, and passed it along until I could hook it in the fold of the neck. By this means I was at length able to extract the head. When this was accomplished, there was very nearly as much difficulty in the passage of the shoulders. Having seized the head and neck firmly, steady traction was made so as almost to dislocate the cervical vertebro, and yet it produced no effect. A blunt hook was then placed in the axilla, and with it one arm passed over the body of the child, and extracted; seizing hold of tine neck and this arm, the remaining shoulder was more readily brought through, and the rest of the body easily followed. The child was small; utrescency was commencing in the funis, but in no other part.

The separation of the placenta was now an object of great anxiety, because if it were adherent, it wonld be extremely difficult to get the hand into the uterus for the purpose of removing it. Fortunately, this did not happen. In about a quarter of an hour after the operation, firm pressure was made by the hand over the fundus of the uterus, by which means the placenta was shot ont of its cavity on the floor. So far this was very satisfactory, as it proved that there mas no want of power in the uterus from exhaustion, and saved us from any apprehension of hremorrhage. The patient was then given a full anodyne, and left in charge of her friends, with strict injunctions that she should not be disturbed.

Having agreed with Mr. Codd on the flan that should be pursued in the future treatment of the case, I returned to town. Mr. Codd kindly undertook to communicate with me from day to day, and I am thus indebted to him for the subsequent report of its progress.

March 13th.-She was free from pain during the night, but slept very little; the pulse 108, sott, and very ensily compressed; tongue soft and moist, covered with white fur'; countenance placid; skin soft and moist; the abdomen soft, slightly tympanitic, but free from pain, except in the iiiac regions. She passed urine freely during the night. The external parts were very slightly swelled, and free from sorcness. She seemed scarcely to have recovered from the shock of the operation. During the day the pulse rose to 120; the tenderness in the iliac regions increased, particularly in the left. She complained of tightness at the epigastrium, and pain on deep pressure over the abdomen. She was bled to sixteen ounces; given calomel and opium, of cach one grain, every three hours; and in the evening, six lecches were applied to each iliac region. The vagina was fomented with poppy injections. 14th. - Spent a restless night, but towards morning slept soundly for an hour. Pulse 132, soft, and compressible; tongue covered with a creamy fur; countenance and spirits good; abdomen very slightly tympanitic, but no tenderness in any part of it. The sense of tightness at the chest has disappeared. The blood drawn was buffed and cupped; the coagulum very firm in a whey-coloured serum. The passages 
were free from tenderness, and very slightly swelled. The same treatment was continued.-Nine o'clock P.M.: Pulse more feeble, 132; tongue very soft and flabby, covered with a great quantity of white fur; twitchings in the fingers; pupils contracted; patient drowsy, but had no sound sleep, nor any desire for food. The abdomen was more tympanitic, but still without pain. The lochia scanty, slimy, and fœetid; no swelling of the external parts. Calomel and opium were discontinued, and the following pills substituted:-Sulphate of quinine, two grains; camphor, one grain; opium, quarter of a grain, every third hour. Decoction of cinchona, two ounces; compound tincture of cardamoms, three drachms; nitric acid, nine minims; water, six ounces: for a mixture. Two tablespoonfuls to be taken every third hour. Turpentine fomentations to the abdomen.

15th.-Had a good night. Pulse still 132, but not so irregular; tongue cleaner and moister. The nervous tremor has disappeared. The abdomen is still tympanitic, but no pain. The bowels have been three times relieved, and she has passed water freely. The lochia still scanty, but less foetid, no appearance of milk in the morning, but towards evening the breasts are becoming full.

16th.-Pulse still 132; tongue cleaner; abdomen less tympanitic; countenance good, without any expression of anxiety; more milk in the breasts; lochia still slightly foetid: she says she feels more comfortable than she possibly could expect. Seven P.M.: has been very easy all day, and has taken arrow-root.

17th.-Pulse still 132; in every other respect improved.

18th.-Pulse 120; tongue soft and moist; abdomen soft, and less tympanitic: says she feels languid: to take a small cup of weak broth every four hours.

19th.- She passed a very good night; pulse 120, soft and compressible; abdomen soft, and free from pain; lochia more abundant, of a good yellow colour; still a little foetid. She felt so well that she sat up in the bed, and, rithout leave, took part of a mutton-chop. She was allowed a sinall quantity of porter, beef-tea, or mutton; and, to correct the fœtor of the discharge, a solution of chloride of lime was injected into the vagina.

Thus far, the progress of the case was most satisfactory; there was every prospect of a perfect recovery, when a new series of symptoms presented themselves that blighted all our hopes.

20th.-She had passed a very restless night from a troublesome cough, and feels an unpleasant weary sensation all over her. She was ordered, morphia, one grain; nitric acid, four minims; infusion of orange-peel, six ounces. Two large tablespoonfuls of the mixture to be taken with each dose of the quinine and camphor.

21st.-Passed the best night she has yet had; pulse 120; the bowels not being relieved, she was ordered an aperient draught, (castor oil, and compound tincture of senna, of each, two drachms; peppermint water, four drachms,) which acted seven or eight times. Mr. Codd was summoned hastily at ten o'clock at night, and found her faint and weak; pulse feeble; abdomen more tympanitic, and some pain in the iliac regions. She was given brandy-and-water, the abdomen fomented with turpentine, and half a grain of morphia every three hours.

22nd.-She slept last night until half-past four o'clock, when she asked for something to take, as she felt pain; she took two cups of tea, said she felt much better, and thought she could sleep a little. Her friends left her about two hours, and were induced to return from hearing her moan heavily. They found her in a dying state. Mr. Codd was again hastily summoned, but she died before his arrival. To explain the cause of death, a post-mortem examination was of the utmost importance. The friends of this patient were greatly opposed to it, more especially as she had herself a great horror of it; and when she feared dissolution, obtained from her sister a promise that she should not be opened. By much persuasion they consented to allow the abdomen to be examined in their presence, but would not snffer the thorax or head to be touched. This was particularly unfortunate, because it has wrapped the cause of death still in mystery. The suspected cause, slough of the cervix uteri, opening into the peritonæum, was proved to be incorrect.

Inspection after death-Countenance placid; extremities moveable and extended; abdomen slightly tympanitic. On opening the peritonæum, the transverse arch of the colon protruded forwards distended with air; the omentum over it was marked with some vessels filled with dark blood; the peritonæum covering the viscera and walls of the abdomen were free from any abnormal appearance excepting at the back of the vagina and neck of the uterus whic was slightly ecchymosed over a space about the size of a crown, but had no appearance of slough, nor was it at all friable; there was no perforation of the peritonseum in any part of it. The uterus was of the ordinary size for that period, the peritongenm covering it pale and glossy; when cut into was rather softer than usual, but very slightly so; the walls were six eighths of an inch thick; its cavity covered with a reddish glairy fluid of the consistence of cream, perfectly inodorous. The pelvis measured in its conjugate axis an inch and a half; the transverse axis, four inches and a half.

The lengthened detail of this case will not permit me to add many observations upon it; I shall therefore merely confine myself to a brief explanation of the principle I kept in riew in the operation, as it will give a reason (I trust, satisfactory) for the time it occupied. Knowing that it was quite uncertain how long the operation would take, I was desirous to avoid as much as possible exposing the patient to protracted and continuous pain. I certainly dreaded the effect of this in producing nervous irritation, and subsequently exhaustion, much more than any danger of inflammation which might arise from delay. So long, therefore, as the action of the uterus continued, extractive force was used only with the pains; when it became more feeble, and the pains were suspended, or came on at longer intervals, the same period of rest was observed between the extractive efforts as when the pains were more powerful. Twice the operation was discontinued, in order to diminish as much as possible any nervous irritation which might cause constitutional shock. In this manner, the delivery, which was commenced on Thursday evening, was not completed until the Friday evening following, and the time actually occupied in the operation, taken collectively, was about seven hours.

\section{ALPHABETICAL NOTICES} OF SUBJECTS CONNECTED WITH

THE TREATMENT OF DYSPEPSIA.

\section{BI ROBERT D I CK, M.D.}

(Continued from p. 334.)

Condiments.-Condiments fulfil two ends. They gratify, during the act of mastication, the gustatory sense, and when justly selected and used, they undoubtedly facilitate, though unconsciously on our parts, the act of digestion. There can be no doubt that, by divine provision, our gustatory predilections, as regards condiments, are, when simple and unsophisticated, precisely those which are nccessary or favourable to perfect chymification. Thus the use of common salt is so universal as to seem the result of a sort of instinct. As chloride of sodium, it serves us as a condiment, in the first place; and afterwards, its hydrochloric acid and soda fulfil various important purposes in primary and secondary assimilation.

There are one or two apparent anomalies in the use of condiments and stimulant food at first sight perplexing, but on reflection easily explicable: knowing that our food is partly respiratory, partly formative, (if we may suggest that word,) and that one of the ends of respiration is to maintain animal temperature, we can account for the predilection, among the inhabitants of northern latitudes, for stimulant food, drink, and condiments. But it seems opposed to nature and consistency that the natives of warm latitudes should make large use, as some of them do, of the most pungent spices. Thus both the indigenous and exotic inhabitants of the East and West Indies, and of other southern countries, employ extensively curries, gingers, tomatos, peppers, mustards, cloves, cinnamon, garlic, \&c., while we should, $\grave{a}$ prior $i$, expect them to use only a mild vegetable diet; and the fact is, that they have a liking for such food, yet, at the same time, make a simultaneous use of the warm condiments referred to; and herein consists the seeming incongruity. The explanation is as follows:-

In these warm countries, the heat, acting strongly on the skin, renders the peripheric circulation full and active, and, in proportion, lessens that of the central organs. Hence some degree of languor and debility in these. The digestive orgaus act feebly, and the secretion of the liver, pancreas, \&c., are less profuse than they should be, ${ }^{*}$ unless stimulated by

* To this cause is owing the frequency of icterus in these countries, whether arising from the non-secretion (suppression) of bile, or to absorption of it, after being secreted. Nor is the occasionally profuse biliary diarrhceas of tropical climates any proot of an habitually greater energy of the liver in these countries than in more temperate ones. For these tormidable biliary discharges are only the consenuences of previous interregna of torpor and deficient secretion, during which the bile has accumulated
to excess in the liver or in the blood. 